\section{第unterian lecturre}

or

\section{THE "TOXAEMIAS" OF PREGNANCY. \\ Delivered at the Royal College of Surgeons, JANUARY 28TH, 1924,}

BY

FRANK COOK, B.Sc., M.B., B.S., F.R.C.S., HUNTERIAN PROFESSOR; OBSTETRIC AND GYNAECOLOGICAL REGISTRAR, GUY'S HOSPITAL; BEIT MEMORIAL RESEARCH FELLOW.

\section{(Abbreviated Report.)}

There has been an illogical tendency to group various disorders of pregnancy under one heading and to attribute their manifestations to a common causal agency. Moreover it has become customary, by the application of the general term " toxaemia," to convey a false impression of knowledge belied by the very diversity of opinion that characterizes our every discussion of the subject. In its literal interpretation, the term is innocent enough; but as commonly employed in this particular connexion it almost imparts that comfortable sense of fatalism which is the most insidious enemy to research. As a necessary preliminary to the scientific study of these problems, I submit that any presupposition of a common etiological factor should be dismissed for the time being; that each form of derangement should be examined strictly on its own merits; and, in certain instances, that each individual case should be regarded as a separate entity.

A tribute is due to the memory of John Lever, sometime obstetrician to Guy's Hospital, who first recorded the association of albuminuria with eclamptic convulsions. The discovery was presumably made under the influence of his contemporary Richard Bright; but it is worthy of note that his treatment differed but little in general from certain much vaunted methods of the present day, and his results were almost equally successful. If we except a death apparently due to other causes, we find that twelve of his thirteen cases recovered. His clinical descriptions are excellent, for he was not obsessed by other than the simplest hypothesis, to which further reference will bo made.

At the present time there is an inclination to assume that noxious elements or toxins tend to arise directly or indirectly from the ovum, and in particular from its growing edge, the placenta, thereby constituting what one might describe as a form of biochemical neoplasia. Alternatively, it is suggested that anaphylactic phenomena are established. Innumerable variations of these theories have been proposed. A different hypothesis has been advanced by Young, who believes that toxins arise from the autolysis of localized areas of placental necrosis, following some form of interference with the maternal blood supply. On first principles one finds this conception more attractive than those in which the living tissues of the ovum are regarded as a potential source of serious disease. Wallis and Williams have suggested that the overproduction of a toxic substance in the corpus luteum is the cause of all the trouble. So great has been the desire to attribute the ills of pregnancy to the agency of toxins that one author has postulated toxic hyperexcitability of the vagus as a satisfactory explanation of pregnancy vomiting.

The Dublin school, whose clinical results are more convincing than their pathological theory in view of the biochemical evidence, appear to imply that excessive metabolic products accompanying the pregnant state subject the excretory organs of the body to an extra strain, which is reinforced by the effects of overeating and defective alimentary elimination; or, as an alternative supposition, that "the food ingested and the toxic elements from the foetus require the same ferments in the blood to neutralize them. The foetal toxin takes some of the elements from the blood which are intended for the digestion of food. This leaves the latter with no feiment, and therefore the food acts as a toxin." "In connexion with this latter view of the situation, the observations of McQuarrie and Whipple with regard to the influence of intestinal obstruction and proteose intoxication on the renal function are of some considerable interest; but $I$ would refer you to an engaging criticism of their work which has recently been published by Paramore.

Paramore maintains, on the basis of clinical and experimental evidence, that the intra-abdominal pressure is definitely raised in pregnancy, and that an exaggerated increase of this pressure determines a toxaemia; but he regards this toxaemia as a purely secondary factor, due to mechanical interference with the capillary circulation of the liver and kidneys, and consequent impairment of their excretory functions.

Vitamins and the ductless glands have naturally been held to blame; and there is indeed one aspect of an endocrine disturbance that may be worthy of attention, although at the moment it is purely speculative. Kark has assumed that eclamptic phenomena represent the effects of an overaction of the pituitary gland, which is normally called into play during the latter periods of pregnancy for obvious physiological purposes. He suggests that this occasional excess of pituitrin in the system leads to contraction of the arterioles in various parts of the body and produces thereby necrotic foci in the placenta, liver, and kidney, and oedema of the brain. We may shortly be in a position to examine the validity of this hypothesis by the application of an extremely delicate test for the detection of pituitrin in the blood.

Quite apart from any potential superabundance of pituitrin it is possible to conceive that a hyperactivity of purposive pressor effects is otherwise induced, whether as a result of circulatory disturbances of reflex or mechanical origin or under the influence of adrenaline or any of the pressor bases. Unfortunately we have no means of detecting the relative quantities, or even the actual existence, of these latter in the general circulation, and for the present we can only hope to observe the vascular phenomena as such.

I do contend, however, with all due deference, that we should confine our investigations to the study of tangible physiological, anatomical, and pathological factors, rather than allow our minds to make use of an imaginary primary specific toxin for the existence of which there is at present no direct scientific evidence whatever.

Biochemical Observations.

Considerable attention has been afforded of recent years to the inorganic constituents of the body under various conditions. This has naturally appealed to those of us who are merely amateur biochemists. Much credit and blame have been attached to the part played by calcium, particularly in the economy of the female. Applying the method of Laidlaw and Payne in determining the total calcium content of the serum in pregnancy, eclampsia, and pregnancy with albuminuria (apart from actual nephritis), I have found no considerable deviation from the normal. Osman and I investigated the acidosis factor in pregnancy. We found, in common with other observers, a reduction in the bicarbonate content of the plasma. No further reduction was found in cases of albuminuria of pregnancy, although in nephritis proper there is frequently a marked decrease in the alkali reserve, associated with phosphate retention and diminution of the calcium content of the serum. In certain cases of eclampsia, however, a definite fall in the plasma bicarbonate was noted; but one has since attributed this to the effects of the convulsions and of labour itself, as corresponding figures have been obtained in the later stages of normal labour, and a particularly low figure in a case of pregnancy accompanied by convulsions due to a definite cerebral lesion. It is now generally agreed that the acidosis factor plays a negligible part in the "toxaemias" of pregnancy. Further reference will be made to this subject under the heading of pregnancy vomiting. As regards the "physiological acidosis". of pregnancy, Marrack has demonstrated a primary reduction of $\mathrm{CO}_{2}$ tension in the blood, and only in a minority of cases an actual alkali deficit, which is presumably due to the excess of base over acid required by the foetus in the later stages of pregnancy. I have been able to detect the characteristic fall in plasma bicarbonate at the earliest 
stages. It is possible that we may eventually find some relationship between the circulatory changes subsequently suggested and the variations in the acid-base balance. On the whole, no conspicuous deviation has been demonstrated by responsible observers in the inorganic constituents of the blood in eclampsia.

De Wesselow, who has published excellent work on this subject, aptly observes that the renal lesions of pregnancy can hardly be attributed to the overstraining of a normal function, since the influence of foetal demands in the ordinary course of events appears actually to diminish the quantities in circulation of such metabolites as require excretion. Even in eclampsia there is not that marked accumulation which is found in certain types of nephritis. Farr and Williams found a variable increase in the nonprotein nitrogen content of the blood in eclampsia; but this was never really excessive, and the extent of the increase bore no definite relation to the severity of the symptoms. As is elsewhere indicated, the ammonia coefficient fallacy has been satisfactorily exploded, and variations in the nitrogen partition of the urine are no longer regarded as an index of that hepatic disorder which may undoubtedly exist. In fact, Nash and Benedict have recently implied that ammonia appearing in the urine is formed by the kidneys and not by the liver. Unfortunately, observations directed to the detection of functional failure on the part of the liver have not hitherto yielded any information of considerable value. ${ }^{*}$ Losee and Van Slyke, whose observations on the alkali reserve we have already confirmed, found no definite increase in the aminonitrogen content of the blood or urine in eclamptic women, and conclude that there is no evidence of defect in the desaminating function of the liver. Alsberg found no impairment of laevulose tolerance in eclampsia, and Tallerman has more recently noted a departure from the normal in only one of three cases.

Dienst suggests that thrombus formation is concerned in the pathology of eclampsia-and it is certainly a factor to which we must give attention. He attributes the tendency to thrombosis, for which there is clinical evidence, to an increased blood content of fibrinogen and fibrin ferment in pregnancy; and states that abnormally high levels are reached in eclampsia. To a certain extent de Wesselow supports this observation, but remarks that much higher levels are reached in cases of pregnancy complicated by infection. Falls has demonstrated a slight increase in the coagulability of the blood in pregnancy, and especially during labour, but regards the variations as being within normal limits.

It must be admitted, after serious consideration of all the available evidence, that biochemical investigations have hitherto failed to elucidate the fundamental factors of these problems. They have reflected the results of derangement, but they have not thrown light on etiological antecedents. It must be remembered, however, that our present-day methods are of a somewhat macroscopic order, and that, although the records may be uninspiring, there is every reason to encourage further biochemical research.

Unfortunately biochemistry is a dangerous ally, and those who fly to its aid have often little knowledge of its actuai values.

\section{Albuminuria of Pregnancy.}

One is inevitably struck by the absence of abnormal features in the average case of albuminuria of pregnancy, with the possible exception of oedema. Although there appears to be only a difference of degree between cases of "symptomless" albuminuria and those which are preeclamptic, the former exhibit remarkably little clinical or biochemical deviation from the physiological standard. This was impressed on my attention in the course of inrestigations on the acidosis factor in normal and abnormal pregnancy. I have further been unable to find any increase whatever in the inorganic phosphate content of the plasma in these cases, such as would afford evidence of renal retention in that respect. De Wesselow found an

E. Since this lecture was delivered, Messrs. Comyns Berkeley and Society of Medicine, with special reference to the detection to the Royal abnormality in eclampsia and the pre-eclamptic state. almost negligible rise in the blood urea content of mild types of albuminuria, although the urea concentration test showed some slight degree of renal impairment. These and other considerations suggested a comparison with various forms of "functional " albuminuria.

In cases of orthostatic albuminuria apparently due to lordosis, Sonne assumed that the two kidneys would not be equally involved, as the left renal vein would be more liable than the right to compression by an anterior curvature of the spine; and he was able to demonstrate by ureteric catheterization a left-sided unilateral albuminuria. Rieser postulated a compression of the left renal vein between the aorta and superior mesenteric artery in cases of visceroptosis associated with lordosis. In view of the mechanical conditions obtaining in late pregnancy, an investigation on these lines seemed therefore to be worthy of pursuit.

I was fortunate in obtaining the expert collaboration of Mr. V. E. Lloyd, who has in each instance undertaken the actual catheterization; but we have been unfortunate in failing to obtain a sufficient number of suitable cases to afford any satisfactory conclusion. Apart from the diffculty of obtaining clinical material, there are various technical difficulties to be encountered. The interior of the bladder may be so distorted in the later stages of pregnancy as to render ureteric catheterization far from easy; and although I have envied the patience, gentleness, and skill which $\mathrm{my}$ colleague has invariably displayed, it has been quite impossible to obtain the desired specimens of urine on every occasion, and our results have thus been limited to somewhat pitiable proportions.

Corresponding specimens of urine were obtained from the left and right kidney respectively in five multigravidae and four primigravidae. The former showed no marked difference in the protein content of the urine from either side. Although there was slightly more on the left than on the right in certain cases-and in no case was this position reversed-the differences were regarded as being within the limits of experimental error. The relative quantities of urine varied considerably, but the variation was not uniform. In none of these cases was there excessive albuminuria. In three of the four primigravidae, however, a definitely asymmetrical excretion of protein was observed: in the first case a heavy cloud on the left and a faint trace on the right; in the second case 20 parts per 1,000 on the left and 6 parts per 1,000 on the right; and in the third case, of which details have already been published, 14 parts per 1,000 on the left and 1.2 parts per 1,000 on the right. The fourth case showed only 0.4 part per 1,000 on either side. There was very little difference in the relative quantities of urine obtained from either ureter over a corresponding period in any of these latter cases. It will be noted that in no case of the whole series was the urine of the right kidney completely free from protein.

In spite of general opinion as expressed in recent years, we are able to suggest, therefore, that mechanical pressure and a resulting hyperaemia of the left kidney exercise a certain etiological influence. At the same time it is indicated by the above observations that this particular factor does not represent the whole matter, since albuminuria was invariably found on the right side in some degree. Rather than attempt to support these experimental data with indirect evidence, which is always so fatally plentiful, we shall propose to continue the investigations as opportunity occurs, and await definite results. In the meantime it may be profitable to examine other possible circulatory alterations with further lines of research in view.

The pregnant uterus may directly produce local venous compression, of which one particular instance has already been suggested. The local pressure effects may be exerted on the vena cava itself or on its branches; further instances are to be found in the varicose veins, haemorrhoids, discoloration of the vulva, and oedema of the lower limbs (apart from renal defect) which commonly accompany pregnancy. It is tempting to postulate, in association with the implied compression of the left renal vein, a circulatory disturbance of the corresponding suprarenal gland, which might explain certain features of eclampsia itself; but until we liave a means of estimating the adrenaline 
content of the blood this conjecture represents the wildest speculation. Fischl was able to produce in animals not only albuminuria but also an actual nephritis by repeated experimental lordosis. Lever hîmself attributed the manifestations of eclampsia to the results of compression of the renal veins by the pregnant uterus.

These influences may be further reinforced by a general augmentation of venous pressure such as would accompany the efforts of labour, vomiting in late pregnancy, convulsions from whatever cause, and even the straining due to constipation. On the other hand, Paramore maintains that increased intra-abdominal pressure directly effects an occlusion of the capillaries in the liver and kidneys.

A further important series of possibilities is suggested by the observations of Pembrey and his fellow workers on the albuminuria which accompanies muscular exercise. This condition is not apparently due to the action of metabolic products, as it is not a marked feature of prolonged periods of muscular work. It was originally attributed to venous congestion due to relative cardiac incompetence. Pembrey and his collaborators, however, found the albuminuria to be preceded by oliguria or anuria, which they believe to represent in turn a purposive renal vasoconstriction. Although the average case of albuminuria of pregnancy is not accompanied by any marked decrease in the output of urine, the more serious cases and the aberrant types mentioned above are commonly characterized by oliguria. In labour we may reasonably expect to find an adjustment similar to that observed in other forms of muscular activity. In pregnancy there are at least two possible factors which might conceivably determine a compensatory vaso-constriction in various parts of the body, including the kidneys and liver: one is a physiological increase in the capacity of the vascular system; another is an increased capacity due to venous obstruction. In this connexion Bolton's well known observations on the effects of experimental narrowing of the inferior vena cava provide invaluable material for meditation.

These and other reflections appear to afford a sufficient indication to resurvey the circulatory conditions as they exist in the pregnant woman. One obvious field of inquiry is provided by a comparison of the rascular features obtaining in quadrupeds, where certain direct pressure effects are presumably eliminated. $U_{p}$ to the present I have had some difficulty in obtaining information in this direction, as few relevant observations seem to have been made on the pregnant animal.

\section{Eclampsia.}

According to the point of view, eclampsia presents a pathological problem of unlimited interest or a train of truly terrifying symptoms. Its most impressive features are the cerebral manifestations and the high blood pressure with which they are commonly associated. The accompanying renal lesions have inevitably suggested a comparison with uraemia, which unfortunately affords us little help. There is even greater variation between different cases of uraemia than there is between different cases of eclampsia. Moreover, as Batty Shaw has pointed out, the term "uraemia" itself is a misnomer; though useful clinically, it implies a state of affairs that is unsupported by experimental evidence. Marrack has justly observed that the disturbances usually classed as anaemic are not necessarily allied with evidence of renal retention or of acidosis. Such disturbances appear to be explained by vascular changes; they are found in patients with high blood pressure and fair renal efficiency. On the other hand, none of the characteristic symptoms may be exhibited in cases with extreme degrees of renal impairment.

It is in general with the former of these types that eclampsia may be appropriately compared, although eclamptic convulsions are occasionally unaccompanied by any marked increase of blood pressure, and although on cursory examination the kidney may show striking evidence of defect. In spite of oliguria and excessive albuminuria, evidence of a serious accumulation of waste roducts in the blood has not been forthcoming as a rule, though it is true that certain of these products might easily have escaped the biochemist's eye.
To attribute all forms of eclampsia to a uniform sequence of pathological events is almost as illogical as to apply a common cause to all the ills of pregnancy, and doubtless' the ienal element assumes a paramount importance in various cases; but it must be admitted that in the majority it would appear to play a secondary part. Certainly brilliant results have been recorded from decapsulation of the kidney in apparently hopeless cases, and it would be absurd to suggest that the renal influence may not predominate; but for the moment we are groping for fundamental etiological factors rather than portraying the manifestations of disease.

Post-mortem and clinical appearances imply a definite derangement of hepatic function. I understand there is corresponding biochemical evidence, but that already published is not convincing; and even were the fact established, it would not necessarily indicate the source of trouble. It is obvious that any satisfactory hypothesis must take into account the liver lesions; and it may be said at once that these are not such as could be explained on the basis of passive venous congestion via the hepatic vein. The influence of toxic bodies in the portal or general circulation, capillary occlusion as suggested by Paramore, or impairment of the afferent blood supply however induced, are all potential factors, though each is highly speculative.

It is easy enough to explain the cerebral symptoms and post-martem findings in the brain on grounds of circulatory disturbances if we consider the possible effects of haemorrhages, thromboses, congestion, and oedema. The excellent results of veratrone administration recorded by Stevens and other's have afforded striking evidence of the influence of blood pressure on eclamptic convulsions; and the occasional good effects of venesection cannot seriously be attributed to the removal of toxins from the circulation. The causes of hyperpiesis have already been discussed; but, at the risk of repetition, it is again suggested that we should to some extent concentrate our attention on the vascular factors of this grave disorder.

\section{"Accidental " Ante-partum Haemorrhage.}

Haemorrhages of this class are commonly designated as "toxaemic." Although the etiology implied may ultimately prove to be correct, the present-day use of the term suggests a somewhat unjustifiable idea of superior information. Fortunately the treatment of the condition rests. on a sound basis provided by general experience. It is due to remark on the excellence of the clinical and pathological records published a few years ago by the late Gordon Ley. Whatever the future may decide as to the validity of his conclusions, the merit of his actual observations will inevitably withstand the test of time. Admittedly this form of haemorrhage is frequently associated with albuminuria, and occasionally with eclampsia; but it is surely illogical to postulate ipso facto a common etiological agency. It is, indeed, obvious that, in the large majority of cases, the albuminuria is not discovered until the haemorrhage is manifest, although it may have preexisted. I have in mind certain instances in which the albuminuria definitely followed the haemorrhage in point of time, and I am equally aware that a reverse order of events may obtain. Systematic ante-natal supervision will determine the common sequence, but this unfortunately will not prove anything. The histological appearances of the affected uterus have not been similarly interpreted by different observers. Although Gordon Ley and others have regarded the oedema, necrosis, and haemorrhage as being due to a common cause (toxaemia), and remarked that the haemorrhages were in fact secondary to the necrosis, it has been alternatively contended that the necrosis is secondary to the haemorrhage.

Dr. James Young has argued that the underlying and primary element is an interference with the maternal blood supply; this may be due to simple mechanical factors determining a separation of the placenta from the uterine wall, or to a blockage of the ovarian or uterine veins from thrombosis, kinking, direct pressure, etc. Without taking into consideration for the moment the further stage of his hypothesis, which attributes any accompanring "toxaemic" symptoms to the autolytic products of secondary placental infarcts, I again submit 
that potential mechanical and vascular factors should be seriously examined. If we reflect on the anatomical relationships of the pelvic and abdominal veins in late pregnancy, the local and general changes in pressure, the possibility of vasomotor disturbance, and that of any increased coagulability of the blood, we shall find a sufticient field for research without the necessity of postulating a "hidden hand." With a growing tendency to apply Caesarean section to the more serious cases of antepartum haemorrhage, our opportunities of direct observation are increasing. Young has compared the appearances in certain cases to those produced by a sudden strangulation of the reins in axial rotation of ovarian cysts; and certainly the aptness of this comparison has been obvious on several occasions in my own experience.

As regards the relationship of the albuminuria, etc., to the haemorrhage, I am not prepared to press any particular hypothesis. The common etiological factor suggesterl by Gordon Ley and others of the same opinion would certainly afford a reasonable explanation were it substantiated in fact, although it is exceptional to find toxaenic symptoms associated with haemorrhages in the earlier months of pregnancy. Young and Miller have adduced, as additional evidence in favour of their theory, a series of cases of placenta pràevia accompanied by albuminuria and even eclampsia; whereas Haffner, after an extensive and careful study, has concluded that there is no direct relationship between albuminuria and placental infarction. Paramore believes that the increased volume of the uterus due to " concealed" haemorrhage so adds to the already raised intra-abdominal pressure as to affect directly the functions of the liver and kidneys. One objection to this eontention is the undoubted association of "aceidental" ante-partum haemorrhage with multiparity, of which a common feature is laxity of the abdominal wall. To say the least, consensus of opinion is not striking; and since the satisfactory nature of the treatment does not demand any special urgency, it behoves ns deliberately and without bias to accumulate definite facts before proceeding to further speculation.

\section{Vomiting of Pregnancy.}

Although no completely satisfactory explanation has hitherto been afforded to account for the milder degrees of nausea and vomiting which so commonly characterize the earlier period of pregnancy, the work of recent years has gone far to elucidate the pathology of those more serious forms of vomiting which have been variously termed " pernicious" or "toxaemic." The former of these expressions is non-committal; but the term "toxaemic" would appear to be especially misleading in this connexion, as every biochemical and histological manifestation scientifically observed can be attributed to the effects of the vomiting itself. I need not review in detail the admirable work of Harding on this subject nor the psychological theory of persistent romiting in pregnancy expounded by Hurst, since these are already well known; but I may be allowed to recount in brief certain observations of my own, although the results are of a negative character.

The work of others, to which further reference will be made, had led me to assume for the time being that the more serious degrees of this condition were due in general to the starvation and dehydration consequent upon the vomiting and lessened intake; at the same time, I had been satisfied by certain definite clinical observations that the ordinary degrees of " morning sickness" or " physiological " vomiting were not entirely attributable to a hysterical factor. The object was therefore to investigate the latter type of case from a biochemical point of view. To provide a working basis sereral factors were taken into consideration. Harding had noted in his earlier papers evidence of ketosis occurring in the mildest cases of nausea, which he attributed to carbohydrate depletion; a lowering of the alkali reserve had been demonstrated even in early pregnancy; and a marked increase in the ammonia-acid ratio had been shown in the urine of more serious cases of pregnancy voniting. It was suggested that this might imply a shortage of phosphate arailable for " buffering" purposes.
In the first place I found that the bicarbonate content of the plasma was not appreciately reduced below the normal pregnancy value, even in cases of moderate severity. This confirms the observations of Losee and $\nabla$ an Slyke in the same direction. It must be remembered, however, that in cases of actual romiting acid is being eliminated via the stomach; this mav account to some extent for the adjustment of the acid-base balance. No evidence was found of any reduction in the blood sugar content; little importance was attached to this observation as the level tends to be maintained even in extreme starvation. As regards the phosphates (which I estimated by Briggs's modification of the Bell Doisy method), it was soon obvious that the inorganic phosphate content of the plasma showed no evidence of depletion; the average figure obtained in cases of vomiting of mild and moderate degree was $3.2 \mathrm{mg}$. phosphorus per $100 \mathrm{c.cm}$. (range 2.35 to 4.0 ). These values may be compared with those obtained by de Wesselow in normal pregnancy and in non-pregnant females. It was further considered, however, that the inorganic phosphate content of the plasma, as in the case of blood sugar, might not justly reflect the phosphorus reserves of the body in general. There are theoretical and particularly practical objections to determinations of a metabolic balance which led me to estimate inistead the "acid-soluble" phosphorus content of the blood corpuscles. For technical reasons, this procedure necessitates estimations of the " acid-soluble" phosphorus content of whole blood and that of its corresponding plasma respectively, together with haematocrit readings in each individual case. The necessary observations and calculations having been made, one again found no evidence of depletion. The average figure obtained in cases of mild and moderate vomiting was $30.6 \mathrm{mg}$. phosphorus per $100 \mathrm{c.cm}$. corpuscles, compared with 29.9 in normal non-pregnant females. (It may perhaps be explained that " acid-soluble" phosphorus includes the phosphorus of inorganic phosphates, together with that of organic substances other than lipoids. The organic factor appears to be contained in the corpuseles, whilst the plasma phosphate is almost entirely inorganic.) The excretion of phosphate during twenty-four hours in a few of the cases under consideration showed no increase; nor was any direct evidence obtained of excessive elimination of acid in the urine. It was further observed that the ammonia-acid ratio showed no definite deviation from the normal-and here $I$ must again remind you that $I$ was investigating cases of only comparatively mild degree. Finally, it became obvious that there was no excretion of "acetone" bodies in the large majority of these cases. Professor Harding has agreed with me that his earlier observations were at fault in this respect.

It is not without interest to note, as indicating the unreliability of unsupported clinical and therapeutic observations, that, pending the results of the above investigation, I treated a very large number of cases of pregnancy vomiting at an ante-natal clinic and elsewhere by administering basic sodium phosphate (in the form of sodii phosphas effervescens) with confidence and with remarkably good results. As these were almost invariably cases of persistent romiting continuing beyond the fifth month of pregnancy, I can only believe that they afforded further evidence of cure by suggestion. The metabolism of phosphorus in pregnancy naturally represents a much more intricate problem than I might appear to have indicated, and negative evidence cannot be conclusive; at the same time there appears to be no indication to proceed further along these particular lines.

It remains to consider for a moment the etiology of the ordinary phenomenon of "morning siekness," and to proceed to an examination of the evidence relating to the more serious conditions of persistent and pernicious vomiting. To explain the former Harding postulated a carbohydrate disturbance occurring in the maternal liver as the primary factor. This derangement of the ketogenic antiketogenic balance essentialiv depends on a shortage of glycogen, due in turn to foetal demands unsatisfied by a sufficiently increased maternal intake, and leads to the production of " acetome" bodies in the blood and urine. Lochhead and Cramer have shown that the presence of the foetus imposes a special demand 
for carbohydrate, and that the pregnant woman has therefore a tendency to pass into a state of specific carbohydrate starvation. But whilst it is freely admitted that these factors assume the utmost importance in continued vomiting, a failure to demonstrate ketosis in the early stages inevitably implies that they are not a primary cause. In fact, we have at the present time, so far as I am aware, no real evidence of biochemical abnormality at the outset of the condition, and still less have we any evidence whatsoever to indicate a specific "toxin." Dr. Hurst has suggested that the "physiological" degrees of nausea and vomiting are reflex in origin, and that the pathological perpetuation and accentuation of the condition are primarily hysterical, the result of auto- and heterosuggestion. These hypotheses are obviously open to doubt if it can be shown that any biochemical deviation from the normal precedes the vomiting, and if any of the manifestations cannot be explained as the result of the vomiting with its consequent starvation and dehydration. Reference has already been made to the misinterpretation of changes in the nitrogen partition of the urine. As a result of persistent vomiting, we have on the one hand a diminished intake of nitrogen and on the other a ketosis, which together can sufficiently account for the high " ammonia coefficients." observed. Underhill and Rand showed that it was possible to ascribe all such changes in pernicious vomiting to starvation, and that the administration of carbohydrate tended to re-establish the normal value. Certainly Gilliatt and Kennaway considered starvation alone to afford an inadequate explanation of the intensity of the changes; but it must be remembered that the disturbance is not so severe as to cause any marked alteration in the acidbase equilibrium. The further data of Harding and Potter are in conformity with the latter observation; they found that, although the excretion of "acetone" bodies in the urine might reach a remarkably high level, their concentration in the blood was not excessive, and not to be compared with that in such cases of diabetes as show a marked depletion of the alkali reserve; nor was the alveolar $\mathrm{CO}_{2}$ generally lowered.

In severe cases an increase in the non-protein nitrogen and uric acid of the blood has been noted. Harding and Drew believe that this is due to the dehydration following continued vomiting, and to an impairment of renal function resulting from the lack of available water. When the anhydraemia and oliguria had been overcome by therapeutic reinforcement of fluids, a reduction of the blood constituents in question was found to accompany the diuresis. Added to the ill effects of carbohydrate starvation in progressive vomiting we must therefore consider the influence of dehydration and its consequences.

As regards the renal impairment, however, we must take into account the further possibility of a mechanical factor on the lines suggested by Paramore. Assuming for the moment that a mechanical or purely vascular factor may determine renal defect in pregnancy, we must admit that violent vomiting would very definitely accentuate its operation, especially in the later stages of pregnancy. I recently had an opportunity of investigating a patient whose various features indicated such an explanation.

There is no reason to believe that every instance of vomiting in pregnancy is attributable to a common cause; the bulk of the evidence is to the contrary. But I do urge that we have at the present time no evidence of a primary toxaemia, and that, generally speaking, the treatment is most emphatically not further starvation.

The nature of my argument precludes a summary of conclusions. If I have suggested anything, it is that we should regard individual cases without preconceived diagnosis or theory, and continue to investigate them with every means at our disposal; that we should accurately record our observations without inductive comment; that we should reconsider and further examine the vascular conditions of pregnancy in general and in detail; and that we should not proclaim the existence and influence of toxic agencies until they have actually been demonstrated.

Without being given to undue optimism in these matters, one feels that the problem of pernicious vomiting has for practical purposes been solved. As regards albuminuria and eclampsia, therapeutic indications still largely rest on a basis of clinical values. My own experience is too small to enable me to add anything of importance to the opinions expressed at the British Congress of Obstetrics and Gynaecology in 1922, to the reports of which I refer you without prejudice. At the same time one would prefer, in the present state of our knowledge, to treat a given case $\mathrm{cn}$ its own merits, rather than in accordance with any prearranged plan, however distinguished its origin. There are very definite objections to the grouping of cases for statistical purposes: not only is it a questionable procedure to subordinate treatment to research, but it is also extremely doubtful whether statistical methods are of any considerable value in elucidating problems that comprise such a multiplicity of elements.

I have already apologized for the poverty of my experimental data. For this I do not entirely accept the blame. In the first place, those of you who have engaged in any combined clinical and biochemical research on cases for whose treatment you may have also been responsible will fully realize the difficulties. Team work is essential in medical research. By "team" one does not imply a committee of individuals similarly, however highly, qualified. The various laboratory methods and the therapeutic technique of the present day each require a special degree of skill and training; and it is almost impossible for any ordinary person to combine efficiency in both respects with the requisite exercise of clinical insight and judgement. At all events the attempt involves an unnecessary expenditure of time and energy.

Secondly, I would refer to the very considerable difficulty that has been experienced in obtaining clinical material and accommodation for cases under investigation. Every available facility has been given by the staff of my own hospital, and I am grateful to others for the occasional opportunity of making observations. But, as Dr. Janet Campbell has emphasized, the limited numbers of obstetric beds in our hospitals afford entirely insufficient scope for teaching purposes; much less is there provision for research. Perhaps it would ultimately lead to a better organization if we clearly and candidly exposed the gaps in our knowledge and at the same time made evident the hopefulness of our task.

[A full list of references and other acknowledgements will be published in a forthcoming edition of the Guy's Hospital Reports.]

\section{Etlemoranda: \\ MEDICAL, SURGICAL, OBSTETRICAL.}

\section{ELEPHANTIASIS NOSTRAS AFTER LYMPHATIC INJURY.}

(With Special Plate.)

Elephantiasis vostras (pseudo-elephantiasis) is sufficiently rare to be always interesting, and this case appears worthy of being reported because of its retarded incidence, the typical appearance of the leg, and the similarity to that produced by filarial infection.

Mrs. W., aged 31, fractured her right ankle when she was 6 years old, and subsequently developed tuberculosis of the bone, which was treated surgically.

When she was 9 years old the glands of the right groin were removed for chronic tuberculous jymphadenitis. The scar of the operation-5 inches in length-lies parallel to and about threequarters of an inch below Poupart's ligament. From the size and quarters of an inch below Poupart's ligament. From the size and carried out, and it is almost certain that either the deep vertical inguinal glands were removed or the lymphatics draining into them injured.

Eight years later-when she was 17-she developed a patch of lupus over the lower part of the tibia. This has since spread all over the leg and foot, as the photograph shows. At the age of 25 over the leg and foot, as the photograph shows. At the age of 25
she complained of "swelling" in the ankle; the oedema has since she complained of " swelling" in the ankle; the oedema has since
spread and now extends to the knee. During the course of the last six years there has been temporary amelioration of the symptoms under treatment--bot baths, thyroid, local applications-but the condition has not been arrested. 